\title{
LINEAR SYSTEMS ON A SPECIAL RATIONAL SURFACE
}

\author{
HENRY K. SCHENCK
}

\begin{abstract}
We study the Hilbert series of two families of ideals generated by powers of linear forms in $\mathbb{K}\left[x_{1}, x_{2}, x_{3}\right]$. Using the results of Emsalem-Iarrobino, we formulate this as a question about fatpoints in $\mathbb{P}^{2}$. This is equivalent to studying the dimension of a linear system on a blow up of $\mathbb{P}^{2}$. We determine the classes of the negative curves, then apply an algorithm of Harbourne to reduce to an effective, nef divisor. Combining Harbourne's results on rational surfaces with $K^{2}>0$ and Riemann-Roch yields a formula for the Hilbert series. For one family of ideals, this proves the $n=3$ case of a conjecture posed by Postnikov and Shapiro "as a challenge to the commutative algebra community" (after this proof was communicated to them, they found a combinatorial proof for all $n$ ). For the second family of ideals, it yields a formula which Postnikov and Shapiro were unable to obtain via combinatorial methods. We conjecture a formula for the minimal free resolution of one family of ideals, and show that a member of the second family of ideals provides a counterexample to a conjecture made by Postnikov and Shapiro in $[20]$.
\end{abstract}

\section{Introduction}

Let $R=\mathbb{K}\left[x_{1}, \ldots, x_{n}\right]$ be a polynomial ring. We consider the following families of $2^{n}-1$ generated ideals:

$$
\begin{gathered}
I_{\phi}=\left\langle x_{1}^{\phi(1)}, \ldots, x_{n}^{\phi(1)},\left(x_{1} x_{2}\right)^{\phi(2)}, \ldots,\left(x_{i_{1}} \cdots x_{i_{r}}\right)^{\phi(r)} \ldots\right\rangle \\
J_{\phi}=\left\langle x_{1}^{\phi(1)}, \ldots, x_{n}^{\phi(1)},\left(x_{1}+x_{2}\right)^{2 \phi(2)}, \ldots,\left(x_{i_{1}}+\cdots+x_{i_{r}}\right)^{r \phi(r)} \ldots\right\rangle,
\end{gathered}
$$

where $\phi$ is either a linear degree function:

$$
\phi(r)=l+k(n-r)>0, k, l \in \mathbb{N} \text { (the positive integers). }
$$

or an almost linear degree function: $\exists k \in \mathbb{N}$ such that

$$
\phi(r)-\phi(r+1)=k \text { or } k+1 \forall r \in\{1, \ldots, n-1\} .
$$

In [19], Postnikov and Shapiro conjecture that for a linear degree function $\phi$, the Hilbert series $P\left(R / I_{\phi}, t\right)$ is equal to the Hilbert series $P\left(R / J_{\phi}, t\right)$. They gave a proof for $n=2$, and for any $n$, when $\phi(r)=n+1-r$. If $\phi$ is almost linear, they observe that the Hilbert series are also often equal, although not always, as there are counterexamples when $n=4$. In this paper, we prove:

Theorem 1.1. If $\phi$ is linear or almost linear, $n=3$, and $\operatorname{char}(\mathbb{K})=0$ or $\operatorname{char}(\mathbb{K})>\sum_{i=1}^{3} \phi(i)-2$, then $P\left(R / I_{\phi}, t\right)=P\left(R / J_{\phi}, t\right)$.

Received March 14, 2004.

Partially supported by NSF grant DMS03-11142 and NSA grant MDA904-0301-0006. 
Theorem 1.1 is proved via algebraic geometry. While none of the previous work on $P\left(R / J_{\phi}, t\right)$ ([19], [20], [22]) made any assumptions on the characteristic of $\mathbb{K}$, some conditions are in fact necessary. We illustrate this in Example 3.2, which shows that in low positive characteristic the conclusion of Theorem 1.1 can fail in very simple cases, and for very simple reasons.

After the proof of Theorem 1.1 was communicated to them [22], Postnikov and Shapiro [20] were able to use combinatorial techniques (the correspondence between parking functions and labeled trees) to give a proof that if $\phi$ is linear then $P\left(R / I_{\phi}, t\right)=P\left(R / J_{\phi}, t\right)$ for any $n$. However, their methods do not handle the almost linear case. They note that except in very special cases a Gröbner basis approach cannot work because the monomial generators do not lie on the boundary of the Newton polytopes of their polynomial deformations. Their motivation for the work was an effort to generalize earlier results [21] on the algebra generated by curvature forms on the generalized flag manifold.

For a monomial ideal $I$, the Taylor resolution ([23], or [4], Exercise 17.11) is a generalization of the Koszul complex which gives an explicit free (generally non-minimal) resolution of $I$. Thus, $P\left(R / I_{\phi}, t\right)$ is known; the interesting case is $R / J_{\phi}$. For a linear degree function, it seems possible that $J_{\phi}$ and $I_{\phi}$ have equal graded Betti numbers; this is consistent with Theorem 1.1. We give an example of an almost linear degree function where $P\left(R / I_{\phi}, t\right)=P\left(R / J_{\phi}, t\right)$, but where the graded Betti numbers differ; this shows that Conjecture 6.10 in [20] is false.

We first use Macaulay's inverse systems approach to relate the dimension of $J_{\phi}$ in degree $j$ to the degree $j$ piece of an ideal $F$ of fatpoints on $\mathbb{P}^{2} . J_{\phi}$ is generated by seven forms, so $F$ is supported at seven points. Following Harbourne, we blow up $\mathbb{P}^{2}$ at the seven points and study a linear system on the resulting surface $X$; because only seven points were blown up we can determine all the negative classes on $X$, which allows us to determine $P\left(R / J_{\phi}, t\right)$. When $n=2, J_{\phi}$ behaves as if the forms were generic. In [6], Fröberg made a conjecture about the behavior of the Hilbert series of an ideal generated by generic forms in $n$ variables, and proved the conjecture when $n=2$; the $n=3$ case was subsequently solved by Anick in [1]. We show that the deviation of the dimension of $\left(J_{\phi}\right)_{j}$ from the dimension of an ideal generated by generic forms of the same degrees is measured by $H^{1}\left(D_{j}\right)$, where $D_{j}$ is a divisor on $X$ corresponding to the degree $j$ piece of a fatpoint ideal on $\mathbb{P}^{2}$.

\section{Linear Forms and Fatpoints}

In [5], Emsalem and Iarrobino proved that there is a close connection between ideals generated by powers of linear forms, and ideals of fatpoints. Let $p_{i}=\left[p_{i 0}\right.$ : $\left.p_{i 1}: \cdots: p_{i n}\right] \in \mathbb{P}^{n}, I\left(p_{i}\right)=\wp_{i} \subseteq R=\mathbb{K}\left[x_{0}, \ldots, x_{n}\right]$, and $L_{p_{i}}=\sum_{j=0}^{n} p_{i_{j}} y_{j}$. Let $\left\{p_{1}, \ldots, p_{m}\right\} \subseteq \mathbb{P}^{n}$ be a set of distinct points. A fatpoints ideal is an ideal of the form

$$
F=\cap_{i=1}^{m} \wp_{i}^{\alpha_{i}} .
$$


Let $S=\mathbb{K}\left[y_{0}, \ldots, y_{n}\right]$, and define an action of $R$ on $S$ by partial differentiation, i.e. $x_{j} \cdot y_{i}=\partial\left(y_{i}\right) / \partial\left(y_{j}\right)$. We think of $S$ both as a ring, isomorphic to $R$, and as an $R$-module. Since $F$ is a submodule of $R$, it acts on $S$, and we can ask what elements of $S$ are annihilated by this action. The set of such elements is denoted by $F^{-1}$. The essential result of Emsalem and Iarrobino is that for $j \gg$ $0,\left(F^{-1}\right)_{j}=\left\langle L_{p_{1}}^{j-\alpha_{1}+1}, \ldots, L_{p_{m}}^{j-\alpha_{m}+1}\right\rangle_{j}$, and that $\operatorname{dim}_{\mathbb{K}}\left(F^{-1}\right)_{j}=\operatorname{dim}_{\mathbb{K}}(R / F)_{j}$. This generalizes the classical Terracini lemma [24], [25], where the $\alpha_{i}$ are all two.

Theorem 2.1. (Emsalem and Iarrobino, [5]) Let $F$ be an ideal of fatpoints:

$$
F=\wp_{1}^{n_{1}+1} \cap \cdots \cap \wp_{m}^{n_{m}+1} .
$$

If $\operatorname{char}(\mathbb{K})=0$ or $\operatorname{char}(\mathbb{K})>j$, then

$$
\left(F^{-1}\right)_{j}= \begin{cases}S_{j} & \text { for } j \leq \max \left\{n_{i}\right\} \\ L_{p_{1}}^{j-n_{1}} S_{n_{1}}+\cdots+L_{p_{m}}^{j-n_{m}} S_{n_{m}} & \text { for } j \geq \max \left\{n_{i}+1\right\}\end{cases}
$$

and

$$
\operatorname{dim}_{\mathbb{K}}\left(F^{-1}\right)_{j}=\operatorname{dim}_{\mathbb{K}}(R / F)_{j}
$$

Suppose we have an ideal generated by powers of linear forms, and for each $j \in \mathbb{N}$, we wish to compute the dimension of

$$
\left\langle L_{p_{1}}^{t_{1}}, \cdots, L_{p_{m}}^{t_{m}}\right\rangle_{j}
$$

Since the $t_{i}$ are fixed, to apply the approach above we fix a degree $j$. Put

$$
F(j)=\wp_{1}^{j-t_{1}+1} \cap \cdots \cap \wp_{m}^{j-t_{m}+1} .
$$

Then

$$
\operatorname{dim}_{\mathbb{K}}\left\langle L_{p_{1}}^{t_{1}}, \cdots, L_{p_{m}}^{t_{m}}\right\rangle_{j}=\operatorname{dim}_{\mathbb{K}}(R / F(j))_{j}
$$

Hence, we will be studying an infinite family of ideals of fatpoints. For additional information on this correspondence we refer to the original paper [5], Geramita [7], or Macaulay [15]; an improved version of Theorem 2.1 is given in [14]. In the next section we apply Theorem 2.1 to study fatpoints ideals in $\mathbb{P}^{2}$ related to linear degree functions, so henceforth we require that the characteristic of our field be zero or greater than $\phi(1)+\phi(2)+\phi(3)-2$.

\section{Blow-ups, Rational Surfaces, and the problem}

Recall now the problem: given a linear degree function

$$
\phi(r)=l+k(n-r)>0, k, l \in \mathbb{N},
$$

determine the Hilbert series of the quotient of $R$ by the ideal generated by (over all tuples)

$$
\left(x_{i_{1}}+\cdots+x_{i_{r}}\right)^{r \phi(r)} .
$$

As we saw in the previous section, this is equivalent to determining the Hilbert function of a family of ideals of fatpoints. In [18] Nagata studied fatpoints in $\mathbb{P}^{2}$, obtaining results for small numbers of points and posing a number of 
conjectures. This continues to be a very active area of research; see [9] or [17] for overviews. Surprisingly, even for a set of points in general position in $\mathbb{P}^{2}$ the Hilbert function is unknown. A formula is conjectured by Segre, Harbourne, Gimigliano, and Hirschowitz, and there has been substantial recent progress: for more, see e.g. [2], [3], [12]. For the remainder of the paper, we specialize to the case of three variables. Given $\phi$, we want to find the Hilbert function of the ideal

$$
\left\langle x^{\phi(1)}, y^{\phi(1)}, z^{\phi(1)},(x+y)^{2 \phi(2)},(x+z)^{2 \phi(2)},(y+z)^{2 \phi(2)},(x+y+z)^{3 \phi(3)}\right\rangle .
$$

Consider the following seven points of $\mathbb{P}^{2}$ :

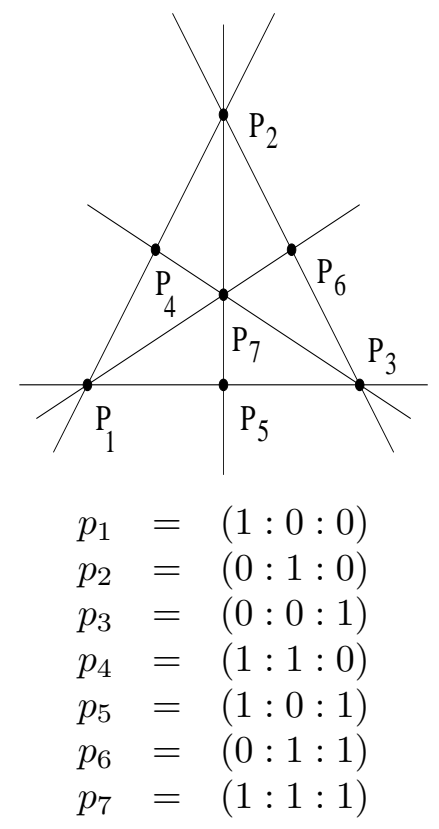

and let $\wp_{i}=I\left(p_{i}\right)$. Define $(a)_{+}=\max \{a, 0\}$. The results of the previous section show that $\operatorname{dim}_{\mathbb{K}}\left(J_{\phi}\right)_{j}=\operatorname{dim}_{\mathbb{K}}(R / F(j))_{j}$, where

$$
F(j)=\left\langle\wp_{1}^{a_{1}} \cap \wp_{2}^{a_{2}} \cap \wp_{3}^{a_{3}} \cap \wp_{4}^{a_{4}} \cap \wp_{5}^{a_{5}} \cap \wp_{6}^{a_{6}} \cap \wp_{7}^{a_{7}}\right\rangle,
$$

and $a_{1}=a_{2}=a_{3}=(j-\phi(1)+1)_{+}, a_{4}=a_{5}=a_{6}=(j-2 \phi(2)+1)_{+}$, and $a_{7}=(j-3 \phi(3)+1)_{+}$. The key to solving this problem is work of Harbourne [10] which shows how to determine the dimension of a linear system on a blow up of $\mathbb{P}^{2}$ at eight or fewer points.

We begin by recalling that there is a correspondence between the graded pieces of an ideal of fatpoints $F$ and the global sections of a certain line bundle on the surface $X$ which is the blow up of $\mathbb{P}^{2}$ at the points. Let $E_{i}$ be the class of the exceptional curve over the point $p_{i}$, and $E_{0}$ the pullback of a line on $\mathbb{P}^{2}$. Put

$$
D_{j}=j E_{0}-\sum_{i=1}^{7} a_{i} E_{i},
$$


with $a_{i}$ as above. The canonical divisor on $X$ is:

$$
K_{X}=-3 E_{0}+\sum_{i=1}^{7} E_{i} .
$$

Since $j>0, h^{2}\left(D_{j}\right)=0$ and Riemann-Roch yields:

$$
\chi\left(D_{j}\right)=h^{0}\left(D_{j}\right)-h^{1}\left(D_{j}\right)=\frac{D_{j}^{2}-D_{j} K_{X}}{2}+1 .
$$

We compute

$$
\begin{aligned}
& \frac{D_{j}^{2}-D_{j} K_{X}}{2}+1= \\
& \quad\left(\begin{array}{c}
j+2 \\
2
\end{array}\right)-3\left(\begin{array}{c}
j+2-\phi(1) \\
2
\end{array}\right)-3\left(\begin{array}{c}
j+2-2 \phi(2) \\
2
\end{array}\right)-\left(\begin{array}{c}
j+2-3 \phi(3) \\
2
\end{array}\right) .
\end{aligned}
$$

Since

$$
F(j)_{j}=H^{0}\left(D_{j}\right)
$$

the dimension of $\left(J_{\phi}\right)_{j}$ is given by:

$$
3\left(\begin{array}{c}
j+2-\phi(1) \\
2
\end{array}\right)+3\left(\begin{array}{c}
j+2-2 \phi(2) \\
2
\end{array}\right)+\left(\begin{array}{c}
j+2-3 \phi(3) \\
2
\end{array}\right)-h^{1}\left(D_{j}\right) .
$$

Notice the connection to Anick's result. $J_{\phi}$ is generated by three forms of degree $\phi(1)$, three forms of degree $2 \phi(2)$, and one form of degree $3 \phi(3)$. Thus, the maximal dimension of $\left\langle J_{\phi}\right\rangle_{j}$ is obtained by multiplying all monomials of degree $j-\phi(1)$ with the generators of degree $\phi(1)$, and similarly for the other generators, so the maximal dimension of $\left\langle J_{\phi}\right\rangle_{j}$ is

$$
3\left(\begin{array}{c}
j-\phi(1)+2 \\
2
\end{array}\right)+3\left(\begin{array}{c}
j-2 \phi(2)+2 \\
2
\end{array}\right)+\left(\begin{array}{c}
j-3 \phi(3)+2 \\
2
\end{array}\right) .
$$

In other words, $\left(J_{\phi}\right)_{j}$ is as large as possible iff $h^{1}\left(D_{j}\right)=0$, i.e. exactly when the divisor $D_{j}$ is nonspecial. The original problem may be restated as follows: for a linear degree function, determine the number of global sections i.e. compute $h^{0}\left(D_{j}\right)$.

Example 3.1. For $n=3$, take $l=3$ and $k=1$. Let $G_{\phi}$ be generated by generic forms of the same degree as the generators of $J_{\phi}$. If $\operatorname{char}(\mathbb{K})=0$ or $\operatorname{char}(\mathbb{K}) \geq 7$, then

$$
\begin{gathered}
P\left(R / G_{\phi}, t\right)=1+3 t+6 t^{2}+10 t^{3}+15 t^{4}+18 t^{5}+19 t^{6}+18 t^{7}+12 t^{8} \\
P\left(R / I_{\phi}, t\right)=P\left(R / J_{\phi}, t\right)=1+3 t+6 t^{2}+10 t^{3}+15 t^{4}+18 t^{5}+19 t^{6}+18 t^{7}+12 t^{8}+6 t^{9} .
\end{gathered}
$$

There are many examples of this type, which is to be expected, since monomial ideals will very rarely have generic Hilbert series. 
Example 3.2. For $n=3$, take $l=1$ and $k=1$. We have that $\phi(1)=3, \phi(2)=$ $2, \phi(3)=1$, so

$$
\begin{gathered}
I_{\phi}=\left\langle x^{3}, y^{3}, z^{3},(x y)^{2},(y z)^{2},(x z)^{2}, x y z\right\rangle . \\
J_{\phi}=\left\langle x^{3}, y^{3}, z^{3},(x+y)^{4},(y+z)^{4},(x+z)^{4},(x+y+z)^{3}\right\rangle .
\end{gathered}
$$

If $\operatorname{char}(\mathbb{K})=2$, then it is obvious that $J_{\phi}$ is in fact minimally generated by $\left\langle x^{3}, y^{3}, z^{3},(x+y+z)^{3}\right\rangle$. A computation shows that in this case

$P\left(R / I_{\phi}, t\right)=1+3 t+6 t^{2}+6 t^{3}$, and $P\left(R / J_{\phi}, t\right)=1+3 t+6 t^{2}+6 t^{3}+4 t^{4}+t^{5}$.

Example 3.3. For $n=3$, take $l=1$ and $k=2$. If $\operatorname{char}(\mathbb{K})=0$ then $J_{\phi}$ is generated by

$$
\begin{array}{rcc}
\left\langle x^{5}, y^{5}, z^{5},(x+y)^{6},(y+z)^{6},(x+z)^{6},(x+y+z)^{3}\right\rangle . \\
j & \chi\left(D_{j}\right) & \operatorname{dim}_{\mathbb{K}}\left(R / J_{\phi}\right)_{j} \\
3 & 9 & 9 \\
4 & 12 & 12 \\
5 & 12 & 12 \\
6 & 6 & 6 \\
7 & -6 & 0
\end{array}
$$

In degree 3 , where $J_{\phi}$ first has a generator, the corresponding fatpoint ideal is the ideal of the point $(1: 1: 1)=\langle x-z, y-z\rangle=F(3)$ and the dimension of $R / F(3)$ in degree 3 is obviously 1 . In degree six we consider the fatpoint ideal

$$
F(6)=\left\langle\wp_{1}^{2} \cap \wp_{2}^{2} \cap \wp_{3}^{2} \cap \wp_{4} \cap \wp_{5} \cap \wp_{6} \cap \wp_{7}^{4}\right\rangle .
$$

To determine the dimension of $(R / F(6))_{6}$, we need to understand effective divisors on $X$. We tackle this in the next section.

\section{Effective Divisors and the linear case}

We first recall some basic terminology, referring to [13] for additional details. Let $X$ be a smooth surface. A divisor $D$ on $X$ is a finite integral combination of irreducible curves on $X$. D is said to be effective if $h^{0}(D)>0$, and numerically effective (nef) if $D \cdot C \geq 0$ for every effective divisor $C$, where - denotes the intersection pairing. In the last section, we saw that Riemann-Roch yields a simple numerical formula for $h^{0}\left(D_{j}\right)-h^{1}\left(D_{j}\right)$; our goal is to determine $h^{0}\left(D_{j}\right)$. A Zariski decomposition of an effective divisor $F$ is a representation $F \sim G+Z$, with $G$ effective and nef, $Z$ effective, and such that $h^{0}(F)=h^{0}(G)$. If $X$ is a blow-up of $\mathbb{P}^{2}$ at a small number of points, then Harbourne gives an algorithm to find a Zariski decomposition in $\S 2$ of [8].

For a smooth projective rational surface $Y$ with $K_{Y}^{2}>0$, Harbourne shows in Theorem 8 of [10] that if $G$ is nef, then $G$ is effective and $h^{1}(G)=h^{2}(G)=0$. Now let $X$ be a blow up of $\mathbb{P}^{2}$ at seven points. Since $K_{X}^{2}=2$, finding a Zariski decomposition $D_{j} \sim G+Z$ and applying Riemann-Roch to $G$ will yield $h^{0}\left(D_{j}\right)$. To obtain a Zariski decomposition of $D_{j}$ in the special case of a blow up at $n \leq 7$ points, test the divisor class $D=D_{j}$ against classes $C$ of negative curves: reduced and irreducible curves with negative self-intersection (in this case, these 
curves generate the monoid $\mathrm{EFF}(\mathrm{X})$ of effective divisor classes, see [11], Remark III.13). Subtract off any such class $C$ meeting $D_{j}$ negatively and continue with $D=D_{j}-C$ either until no negative curves meet $D$ negatively, or until $D \cdot E_{0}<0$. One of these two outcomes is guaranteed to occur. If the former happens, $D_{j}$ was effective and we take $G=D$; if the latter, then $D_{j}$ was not effective to begin with. So the first task is to determine the negative curves.

For seven general points in $\mathbb{P}^{2}$, any negative curve is a -1 curve. These consist of the blow ups of the points, the proper transforms of lines through pairs of points, proper transforms of conics through any five points, and proper transforms of cubics through six points, with a double point at the seventh (see [16], Theorem 26.2). As the points specialize, these -1 curves can become reducible, but the classes of their irreducible components generate $\operatorname{EFF}(\mathrm{X})$. As in the previous section, let $E_{i}$ be the exceptional divisor over a point $p_{i}$, and $E_{0}$ the class of the pullback of a line.

Lemma 4.1. The negative curves on $X$ are the $E_{i}, i \neq 0$, the six -2 curves corresponding to lines through three collinear points, and the three -1 curves corresponding to lines through the pairs $\left\{p_{4}, p_{5}\right\},\left\{p_{4}, p_{6}\right\},\left\{p_{5}, p_{6}\right\}$ :

$$
\begin{aligned}
C_{124} & =E_{0}-E_{1}-E_{2}-E_{4} \\
C_{135} & =E_{0}-E_{1}-E_{3}-E_{5} \\
C_{236} & =E_{0}-E_{2}-E_{3}-E_{6} \\
C_{167} & =E_{0}-E_{1}-E_{6}-E_{7} \\
C_{257} & =E_{0}-E_{2}-E_{5}-E_{7} \\
C_{347} & =E_{0}-E_{3}-E_{4}-E_{7} \\
C_{45} & =E_{0}-E_{4}-E_{5} \\
C_{46} & =E_{0}-E_{4}-E_{6} \\
C_{56} & =E_{0}-E_{5}-E_{6}
\end{aligned}
$$

Proof. First, observe that $-K_{X}$ is nef. This follows since if a divisor $D \sim$ $\sum_{a_{i} \geq 0} a_{i} C_{i}$ with $C_{i}$ effective and irreducible and $D \cdot C_{i} \geq 0$, then $D$ is nef: if not, then $D \cdot F<0$ for some effective curve $F$, so $D \cdot F^{\prime}<0$ for some irreducible component $F^{\prime}$ of $F$. So $F^{\prime}$ meets a summand of $D$ negatively; since the summand is irreducible, it must be a multiple of $F^{\prime}$. This means a summand of $D$ meets $D$ negatively, a contradiction. In particular, $-K_{X}$ is nef since

$$
-K_{X}=C_{167}+C_{236}+C_{45}+E_{6} .
$$

Any curve $C$ must satisfy the genus formula $C^{2}+C \cdot K_{X}=2 g_{C}-2$. Since $-K_{X}$ is nef, $-K_{X} \cdot C \geq 0$; and $g_{C}$ is also non-negative, so the possibilities for negative curves are $C^{2}=-1=K_{X} \cdot C$ and $C^{2}=-2, K_{X} \cdot C=0$. In both cases, there are only finitely many solutions (see [16], 25.5.3, 26.1); we want those which are not forced by Bezout to be reducible, which are those listed above.

We return to Example 3.3:

$$
D_{6}=6 E_{0}-2 E_{1}-2 E_{2}-2 E_{3}-E_{4}-E_{5}-E_{6}-4 E_{7} .
$$


We compute that $D_{6} \cdot C_{167}<0,\left(D_{6}-C_{167}\right) \cdot C_{257}<0$, and $\left(D_{6}-C_{167}-C_{257}\right)$. $C_{347}<0$. Removing $C_{167}+C_{257}+C_{347}$ from $D_{6}$ yields an effective, nef divisor:

$$
G=3 E_{0}-E_{1}-E_{2}-E_{3}-E_{7} .
$$

The rest is easy: $G^{2}=5, G K=-5$, so $h^{0}(G)=6$. Similar computations show that this $J_{\phi}$ behaves generically.

This example illustrates the crucial idea for the general case. Observe that the classes $C_{i j k}$ corresponding to lines with three collinear points are orthogonal.

$D_{j}=j E_{0}-\sum_{i=1}^{7} a_{i} E_{i}$ meets $C_{124}$ negatively iff

$$
j<a_{1}+a_{2}+a_{4}
$$

and since $C_{124}, C_{135}, C_{236}$ each intersect $D_{j}$ with the same multiplicity, anytime that we remove one class, we need to remove all three classes. Of course, this depends on the fact that we began with $a_{1}=a_{2}=a_{3}$ and $a_{4}=a_{5}=a_{6}$. In the same vein,

$$
D_{j} \cdot C_{167}<0 \text { iff } j<a_{1}+a_{6}+a_{7},
$$

and the curves $C_{167}, C_{257}, C_{347}$ meet $D_{j}$ with the same multiplicity, so again, when we remove one of these three, we have to remove all of them. The point is that both of these reductions preserve the equality of the coefficients of $\left\{E_{1}, E_{2}, E_{3}\right\}$ and of $\left\{E_{4}, E_{5}, E_{6}\right\}$. For the rest of the section, let $\phi$ be a linear degree function:

$$
\begin{array}{llc}
\phi(1) & = & l+2 k \\
\phi(2) & = & l+k \\
\phi(3) & = & l
\end{array}
$$

for some positive integers $l, k$; recall the notation $(a)_{+}=\max \{a, 0\}$.

Lemma 4.2. $D_{j}$ meets $C_{124}, C_{135}, C_{236}$ negatively iff $j \geq \phi(1)+\phi(2)-1$. Put

$$
t_{1}=(j-2 l-3 k+2)_{+}=(j-\phi(1)-\phi(2)+2)_{+}
$$

Then

$$
D_{j}^{\prime}=\left(j-3 t_{1}\right) E_{0}-\sum_{i=1}^{3}\left(a_{i}-2 t_{1}\right) E_{i}-\sum_{i=4}^{6}\left(a_{i}-t_{1}\right) E_{i}-a_{7} E_{7}
$$

meets $C_{124}, C_{135}, C_{236}$ non-negatively, and has $h^{0}\left(D_{j}\right)=h^{0}\left(D_{j}^{\prime}\right)$.

Proof.

$$
\begin{aligned}
& D_{j} \cdot C_{124}=j-2(j-\phi(1)+1)_{+}-(j-2 \phi(2)+1)_{+} \\
& =j-2(j-l-2 k+1)_{+}-(j-2 l-2 k+1)_{+} \\
& j \leq l+2 k-1 \quad \Rightarrow \quad D_{j} \cdot C_{124}=\quad j \geq 0 \\
& l+2 k-1<j \leq 2 l+2 k-1 \Rightarrow D_{j} \cdot C_{124}=-j+2 l+4 k-2 \geq 0 \\
& 2 l+2 k-1<j \quad \Rightarrow \quad D_{j} \cdot C_{124}=-2 j+4 l+6 k-3
\end{aligned}
$$


So, $D_{j} \cdot C_{124}<0$ iff $2 j \geq 4 l+6 k-2$ iff $j \geq 2 l+3 k-1=\phi(1)+\phi(2)-1$, and removing $t_{1}$ copies of $C_{124}+C_{135}+C_{236}$ from $D_{j}$ yields a divisor $D_{j}^{\prime}$ which meets the classes $C_{124}, C_{135}, C_{236}$ non-negatively, and has $h^{0}\left(D_{j}\right)=h^{0}\left(D_{j}^{\prime}\right)$.

Lemma 4.3. $D_{j}$ meets $C_{167}, C_{257}, C_{347}$ negatively iff $j \geq 2 \phi(2)+\phi(3)-1$. Put

$$
t_{2}=(j-3 l-2 k+2)_{+}=(j-2 \phi(2)-\phi(3)+2)_{+}
$$

Then

$$
D_{j}^{\prime \prime}=\left(j-3 t_{2}\right) E_{0}-\sum_{i=1}^{6}\left(a_{i}-t_{2}\right) E_{i}-\left(a_{7}-3 t_{2}\right) E_{7}
$$

meets $C_{167}, C_{257}, C_{347}$ non-negatively, and has $h^{0}\left(D_{j}\right)=h^{0}\left(D_{j}^{\prime \prime}\right)$.

Proof.

$$
\begin{aligned}
D_{j} \cdot C_{167} & =j-(j-\phi(1)+1)_{+}-(j-2 \phi(2)+1)_{+}-(j-3 \phi(3)+1)_{+} \\
& =j-(j-l-2 k+1)_{+}-(j-2 l-2 k+1)_{+}-(j-3 l+1)_{+}
\end{aligned}
$$

We have to analyze three possible cases:

$$
\begin{gathered}
l+2 k-1 \leq 2 l+2 k-1 \leq \\
l+2 k-1 \leq 3 l-1 \\
3 l-1 \leq l+2 k-1 \leq 2 l+2 k-1 \\
\leq 1 \leq 2 k-1
\end{gathered}
$$

In the first case, we find

$$
\begin{array}{clccc}
0 \leq j \leq l+2 k-1 & \Rightarrow & D_{j} \cdot C_{167} & = & j \geq 0 \\
l+2 k-1<j \leq 2 l+2 k-1 & \Rightarrow & D_{j} \cdot C_{167} & = & l+2 k-1 \geq 0 \\
2 l+2 k-1<j \leq 3 l-1 & \Rightarrow & D_{j} \cdot C_{167} & = & -j+3 l+4 k-2 \geq 0 \\
3 l-1<j & \Rightarrow & D_{j} \cdot C_{167} & = & -2 j+6 l+4 k-3
\end{array}
$$

The second case differs for the values of $j$ between $l+2 k-1$ and $2 l+2 k-1$ :

$$
\begin{aligned}
& l+2 k-1<j \leq 3 l-1 \quad \Rightarrow \quad D_{j} \cdot C_{167}=l+2 k-1 \geq 0 \\
& 3 l-1<j \leq 2 l+2 k-1 \Rightarrow D_{j} \cdot C_{167}=-j+4 l+2 k-2 \geq 0,
\end{aligned}
$$

and the third case differs for the values of $j$ between $3 l-1$ and $2 l+2 k-1$ :

$$
\begin{aligned}
& 3 l-1<j \leq l+2 k-1 \quad \Rightarrow \quad D_{j} \cdot C_{167}=3 l-1 \geq 0 \\
& l+2 k-1<j \leq 2 l+2 k-1 \Rightarrow D_{j} \cdot C_{167}=-j+4 l+2 k-2 \geq 0 .
\end{aligned}
$$

In all three cases, we find $-2 j+6 l+4 k-3<0$ iff $2 j \geq 6 l+4 k-2$ iff $j \geq$ $3 l+2 k-1=2 \phi(2)+\phi(3)-1$.

Theorem 4.4. Let $\phi$ be a linear degree function, and let $D_{j}=j E_{0}-\sum_{i=1}^{7} a_{i} E_{i}$, where

$$
\begin{array}{ccc}
a_{1}=a_{2}=a_{3} & =(j-\phi(1)+1)_{+} \\
a_{4}=a_{5}=a_{6} & =(j-2 \phi(2)+1)_{+} \\
a_{7} & =(j-3 \phi(3)+1)_{+}
\end{array}
$$

Put

$$
\begin{aligned}
& t_{1}=(j-\phi(1)-\phi(2)+2)_{+} \\
& t_{2}=(j-2 \phi(2)-\phi(3)+2)_{+}
\end{aligned}
$$


Then

$G=\left(j-3 t_{1}-3 t_{2}\right) E_{0}-\sum_{i=1}^{3}\left(a_{i}-2 t_{1}-t_{2}\right) E_{i}-\sum_{i=4}^{6}\left(a_{i}-t_{1}-t_{2}\right) E_{i}-\left(a_{7}-3 t_{2}\right) E_{7}$

is a divisor with $h^{0}\left(D_{j}\right)=h^{0}(G)$. If $j \geq \phi(1)+\phi(2)+\phi(3)-2$, then $h^{0}(G)=0$, and if $j<\phi(1)+\phi(2)+\phi(3)-2$, then $G$ is an effective, nef divisor with

$$
\begin{gathered}
h^{0}(G)=\left(\begin{array}{c}
j+2 \\
2
\end{array}\right)-3\left(\begin{array}{c}
j+2-\phi(1) \\
2
\end{array}\right)-3\left(\begin{array}{c}
j+2-2 \phi(2) \\
2
\end{array}\right)-\left(\begin{array}{c}
j+2-3 \phi(3) \\
2
\end{array}\right) \\
+6\left(\begin{array}{c}
j+2-\phi(1)-\phi(2) \\
2
\end{array}\right) \\
+6\left(\begin{array}{c}
j+2-2 \phi(2)-\phi(3) \\
2
\end{array}\right)
\end{gathered}
$$

The first row is precisely the dimension when $h^{1}\left(D_{j}\right)=0$, while the second row is zero iff $t_{1}<2$ and the third row is zero iff $t_{2}<2$.

Proof. Using Lemma 4.2 and Lemma $4.3, D_{j}$ can be reduced to a divisor $G$ which meets the six -2 curves non-negatively; since the -2 curves are mutually orthogonal the order of the reduction is irrelevant. Now, if $j \geq \phi(1)+\phi(2)+$ $\phi(3)-2=3 l+3 k-2$, then $(j-2 l-3 k+2)_{+}=j-2 l-3 k+2$ and $(j-3 l-2 k+2)_{+}=$ $j-3 l-2 k+2$. So $G \cdot E_{0}=-5 j+15 l+15 k-12$. Our assumption that $j \geq 3 l+3 k-2$ the implies that $G \cdot E_{0}<0$. In particular, $h^{0}(G)=h^{0}\left(D_{j}\right)=0$.

Now assume $j<\phi(1)+\phi(2)+\phi(3)-2=3 l+3 k-2$. To show that $G$ is effective and nef, we need to show that $G \cdot C_{i j} \geq 0$ and $G \cdot E_{i} \geq 0$. Since the curves $C_{45}, C_{46}, C_{56}$ intersect $G$ with the same multiplicity, to show that $G \cdot C_{i j} \geq 0$ it suffices to show $G \cdot C_{45} \geq 0$. We have that

$$
\begin{array}{ccc}
G \cdot C_{45} & = & j-3 t_{1}-3 t_{2}-2\left(a_{4}-t_{1}-t_{2}\right) \\
& = & j-t_{1}-t_{2}-2 a_{4} \\
& = & j-(j-2 l-3 k+2)_{+}-(j-3 l-2 k+2)_{+}-2(j-2 l-2 k+1)_{+}
\end{array}
$$

There are two cases to consider, depending on the relative values of $l, k$. First, suppose $3 l+2 k-2 \leq 2 l+3 k-2$.

$$
\begin{aligned}
& 0 \leq j \leq 2 l+2 k-1 \quad \Rightarrow G \cdot C_{45} \quad=\quad j \geq 0 \\
& 2 l+2 k-1<j \leq 3 l+2 k-2 \Rightarrow G \cdot C_{45}=-j+4 l+4 k-2 \geq 0 \\
& 3 l+2 k-2<j \leq 2 l+3 k-2 \Rightarrow G \cdot C_{45}=-2 j+7 l+6 k-4 \geq 0 \\
& 2 l+3 k-2<j<3 l+3 k-2 \Rightarrow G \cdot C_{45}=-3 j+9 l+9 k-6 \geq 0
\end{aligned}
$$

The analysis if $2 l+3 k-2 \leq 3 l+2 k-2$ differs only for $j$ such that:

$$
\begin{aligned}
& 2 l+2 k-1<j \leq 2 l+3 k-2 \quad \Rightarrow \quad G \cdot C_{45}=-j+4 l+4 k-2 \geq 0 \\
& 2 l+3 k-2<j \leq 3 l+2 k-2 \quad \Rightarrow \quad G \cdot C_{45}=-2 j+6 l+7 k-4 \geq 0
\end{aligned}
$$


Next, we consider $G \cdot E_{i}$. Because $E_{1}, E_{2}, E_{3}$ and $E_{4}, E_{5}, E_{6}$ appear with the same multiplicity in $G$, it suffices to test $G$ against the classes $E_{1}, E_{4}$ and $E_{7}$.

$$
\begin{array}{rlc}
G \cdot E_{1} & = & a_{1}-2 t_{1}-t_{2} \\
& =(j-\phi(1)+1)_{+}-2(j-\phi(1)-\phi(2)+2)_{+}-(j-2 \phi(2)-\phi(3)+2)_{+} \\
& = & (j-l-2 k+1)_{+}-2(j-2 l-3 k+2)_{+}-(j-3 l-2 k+2)_{+}
\end{array}
$$

First, suppose $2 l+3 k-2 \leq 3 l+2 k-2$.

$$
\begin{aligned}
& 0 \leq j \leq l+2 k-1 \quad \Rightarrow G \cdot E_{1}=\quad j \geq 0 \\
& l+2 k-1<j \leq 2 l+3 k-2 \Rightarrow G \cdot E_{1}=j-l-2 k+1 \geq 0 \\
& 2 l+3 k-2<j \leq 3 l+2 k-2 \Rightarrow G \cdot E_{1}=-j+3 l+4 k-3 \geq 0 \\
& 3 l+2 k-2<j<3 l+3 k-2 \Rightarrow G \cdot E_{1}=-2 j+6 l+6 k-5 \geq 0
\end{aligned}
$$

If $3 l+2 k-2 \leq 2 l+3 k-2$, then the values differ for $j$ such that:

$$
\begin{aligned}
& l+2 k-1<j \leq 3 l+2 k-2 \Rightarrow G \cdot E_{1}=j-l-2 k+1 \geq 0 \\
& 3 l+2 k-2<j \leq 2 l+3 k-2 \Rightarrow G \cdot E_{1}=\quad 2 l-1 \geq 0
\end{aligned}
$$

In both cases, we see that $G \cdot E_{1}<0$ iff $2 j>6 l+6 k-5$ iff $j \geq 3 l+3 k-2=$ $\phi(1)+\phi(2)+\phi(3)-2$. The analysis for the classes $E_{4}$ and $E_{7}$ runs along the same lines, with the same result: $G \cdot E_{4}<0$ iff $j \geq 3 l+3 k-2=\phi(1)+\phi(2)+\phi(3)-2$ iff $G \cdot E_{7}<0$.

Thus, if $j<\phi(1)+\phi(2)+\phi(3)-2$, then Harbourne's results imply that $G$ is an effective, nef divisor, with $h^{1}(G)=h^{2}(G)=0$, and $h^{0}(G)=h^{0}\left(D_{j}\right)$. This reduces the computation of $h^{0}\left(D_{j}\right)$ to a simple numerical calculation, the point being that for $G, h^{1}(G)=h^{2}(G)=0$, so:

$$
h^{0}(G)=\chi(G)=\frac{G^{2}-G K_{X}}{2}+1 .
$$

When $t_{1}=1$, the second row of the formula does not contribute to $h^{0}(G)$ because if $t_{1}=1$, then $D_{j}^{\prime}=D_{j}-C_{124}-C_{135}-C_{236}$ and a calculation shows that

$$
D_{j}^{2}-D_{j} \cdot K=D_{j}^{\prime 2}-D_{j}^{\prime} \cdot K
$$

This stems from the choice of a degree function which is linear; a similar observation pertains when $t_{2}=1$.

In [10], Harbourne works over an algebraically closed field $\mathbb{K}$, but as long as $\mathbb{K}$ contains the coordinates of the points being blown up, this hypothesis is unnecessary. This is because the rank of the matrix which computes the dimension of the space of global sections is the same over $\mathbb{K}$ or $\overline{\mathbb{K}}$. However, to apply the results of Section 2, we need to assume that $\operatorname{char}(\mathbb{K})=0$ or $\operatorname{char}(\mathbb{K})>\sum_{i=1}^{3} \phi(i)-2$. In this case, the theory of inverse systems tells us that $\operatorname{dim}_{\mathbb{K}} H^{0}\left(D_{j}\right)=\operatorname{dim}_{\mathbb{K}}\left(R / J_{\phi}\right)_{j}$. A simple computation translates this data into the Hilbert series: 
Corollary 4.5. For $n=3$ and $\phi$ linear, if $\operatorname{char}(\mathbb{K})>\phi(1)+\phi(2)+\phi(3)-2$ or $\operatorname{char}(\mathbb{K})=0$, then $P\left(R / J_{\phi}, t\right)=$

$$
\frac{1-3 t^{\phi(1)}-3 t^{2 \phi(2)}-t^{3 \phi(3)}+6 t^{\phi(1)+\phi(2)}+6 t^{2 \phi(2)+\phi(3)}-6 t^{\phi(1)+\phi(2)+\phi(3)}}{(1-t)^{3}} .
$$

Computing the Hilbert series of $R / I_{\phi}$ via the Taylor resolution mentioned earlier and comparing with the result above proves Theorem 1.1 for a linear degree function. While it may be possible to obtain a better lower bound on $\operatorname{char}(\mathbb{K})$, Example 3.2 shows that this bound is sometimes tight.

\section{Almost linear degree functions}

A degree function $\phi$ (with values in $\mathbb{N}$ ) is called almost linear if there is a $k \in \mathbb{N}$ such that

$$
\phi(r)-\phi(r+1)=k \text { or } k+1 \forall r \in\{1, \ldots, n-1\} .
$$

When $n=3$, an almost linear degree function satisfies the same key property satisfied by a linear degree function: if $D_{j}$ is effective, then it can be reduced to an effective, nef divisor using only the classes $C_{i j k}$. For an almost linear degree function, the Hilbert series of $R / J_{\phi}$ will have two possible forms, depending on the difference vector $\Delta=(\phi(1)-\phi(2), \phi(2)-\phi(3))$.

Lemma 5.1. Let $\phi$ be an almost linear degree function. Then $D_{j}$ meets $C_{124}, C_{135}, C_{236}$ negatively iff $j \geq \phi(1)+\phi(2)-1$. Put

$$
t_{1}=(j-\phi(1)-\phi(2)+2)_{+}
$$

Then

$$
D_{j}^{\prime}=\left(j-3 t_{1}\right) E_{0}-\sum_{i=1}^{3}\left(a_{i}-2 t_{1}\right) E_{i}-\sum_{i=4}^{6}\left(a_{i}-t_{1}\right) E_{i}-a_{7} E_{7}
$$

meets $C_{124}, C_{135}, C_{236}$ non-negatively, and has $h^{0}\left(D_{j}\right)=h^{0}\left(D_{j}^{\prime}\right)$.

Proof. We have to consider two cases. First, suppose $\Delta=(k+1, k)$. For some $l \in \mathbb{N}$ we have $\phi(3)=l$, so $\phi(2)=l+k$ and $\phi(1)=l+2 k+1$.

$$
\begin{aligned}
& D_{j} \cdot C_{124}=j-2(j-\phi(1)+1)_{+}-(j-2 \phi(2)+1)_{+} \\
& =j-2(j-l-2 k)_{+}-(j-2 l-2 k+1)_{+} \\
& 0 \leq j \leq l+2 k \quad \Rightarrow \quad D_{j} \cdot C_{124} \quad=\quad j \geq 0 \\
& l+2 k<j \leq 2 l+2 k-1 \Rightarrow D_{j} \cdot C_{124}=-j+2 l+4 k \geq 0 \\
& 2 l+2 k-1<j \quad \Rightarrow \quad D_{j} \cdot C_{124}=-2 j+4 l+6 k-1
\end{aligned}
$$

So $D_{j} \cdot C_{124}<0$ iff $2 j \geq 4 l+6 k$ iff $j \geq 2 l+3 k=\phi(1)+\phi(2)-1$. Now suppose $\Delta=(k, k+1)$, and let $\phi(3)=l$, so $\phi(2)=l+k+1$ and $\phi(1)=l+2 k+1$.

$$
\begin{aligned}
D_{j} \cdot C_{124} & =j-2(j-\phi(1)+1)_{+}-(j-2 \phi(2)+1)_{+} \\
& =j-2(j-l-2 k)_{+}-(j-2 l-2 k-1)_{+}
\end{aligned}
$$


Proceeding as in the previous case, we find $D_{j} \cdot C_{124}<0$ iff $2 j \geq 4 l+6 k+2$ iff $j \geq 2 l+3 k+1=\phi(1)+\phi(2)-1$.

Theorem 5.2. Let $\phi$ be an almost linear degree function; define $a_{i}$ and $D_{j}$ as in the previous section. Put

$$
t_{1}=(j-\phi(1)-\phi(2)+2)_{+}, t_{2}=(j-2 \phi(2)-\phi(3)+2)_{+}
$$

Then

$G=\left(j-3 t_{1}-3 t_{2}\right) E_{0}-\sum_{i=1}^{3}\left(a_{i}-2 t_{1}-t_{2}\right) E_{i}-\sum_{i=4}^{6}\left(a_{i}-t_{1}-t_{2}\right) E_{i}-\left(a_{7}-3 t_{2}\right) E_{7}$

is a divisor with $h^{0}\left(D_{j}\right)=h^{0}(G)$. If $j \geq \phi(1)+\phi(2)+\phi(3)-2$, then $h^{0}(G)=0$, and if $j<\phi(1)+\phi(2)+\phi(3)-2$, then $G$ is effective and nef, with $h^{0}\left(D_{j}\right)=$ $h^{0}(G)=$

$$
\begin{gathered}
\left(\begin{array}{c}
j+2 \\
2
\end{array}\right)-3\left(\begin{array}{c}
j+2-\phi(1) \\
2
\end{array}\right)-3\left(\begin{array}{c}
j+2-2 \phi(2) \\
2
\end{array}\right)-\left(\begin{array}{c}
j+2-3 \phi(3) \\
2
\end{array}\right) \\
+6\left(\begin{array}{c}
j+2-\phi(1)-\phi(2) \\
2
\end{array}\right) \\
+3\left(\begin{array}{c}
j+2-\phi(3)-2 \phi(2) \\
2
\end{array}\right)+3\left(\begin{array}{c}
j+2-\phi(1)-2 \phi(3) \\
2
\end{array}\right)
\end{gathered}
$$

The first row is precisely the dimension when $h^{1}\left(D_{j}\right)=0$, while the second row is zero iff $t_{1}<2$ and the third row is zero iff $t_{2}<1$.

Proof. First, suppose $\Delta=(k, k+1)$, so for some $l \in \mathbb{N}$ we can write $\phi(3)=l$, $\phi(2)=l+k+1$ and $\phi(1)=l+2 k+1$.

$$
\begin{array}{rlc}
D_{j} \cdot C_{167} & =j-(j-\phi(1)+1)_{+}-(j-2 \phi(2)+1)_{+}-(j-3 \phi(3)+1)_{+} \\
& =j-(j-l-2 k)_{+}-(j-2 l-2 k-1)_{+}-(j-3 l+1)_{+}
\end{array}
$$

We have to analyze three possible cases

$$
\begin{gathered}
l+2 k \leq 2 l+2 k+1 \leq c \\
l+2 k \leq 3 l-1 \\
3 l-1 \leq l+2 k \leq 2 l+2 k+1 \\
\leq \quad l+2 k+1
\end{gathered}
$$

Working through the possibilities, we find $D_{j} \cdot C_{167}<0$ iff $j \geq 3 l+2 k+1=$ $\phi(1)+2 \phi(3)$. So removing $t_{2}=(j-\phi(1)-2 \phi(3)+1)_{+}$copies of $C_{167}+C_{257}+C_{347}$ yields a divisor which meets each of the classes $\left\{C_{167}, C_{257}, C_{347}\right\}$ non-negatively; and applying Lemma 5.1 results in a divisor $G$ which meets all the -2-curves nonnegatively. If $j \geq \phi(1)+\phi(2)+\phi(3)-2=3 l+3 k$, then $G \cdot E_{0}=-5 j+15 l+15 k$. So if $j>\phi(1)+\phi(2)+\phi(3)-2, G \cdot E_{0}<0$ and $h^{0}(G)=0$. If $j=\phi(1)+\phi(2)+\phi(3)-2$, then $G=-E_{4}-E_{5}-E_{6}+E_{7}$. Since $G \cdot C_{45}<0$, the algorithm tells us to further reduce $G$ by removing $C_{45}$, which leaves the divisor $-E_{0}-E_{6}+E_{7}$, hence $h^{0}(G)=0$. 
Now suppose $j<\phi(1)+\phi(2)+\phi(3)-2=3 l+3 k$. As in the proof of Theorem 4.4 , to show that $G$ is effective and nef it suffices to show that $G$ meets the classes $\left\{C_{45}, E_{1}, E_{4}, E_{7}\right\}$ non-negatively.

$$
\begin{array}{rcc}
G \cdot C_{45} & = & j-3 t_{1}-3 t_{2}-2\left(a_{4}-t_{1}-t_{2}\right) \\
& = & j-t_{1}-t_{2}-2 a_{4} \\
& = & j-(j-2 l-3 k)_{+}-(j-3 l-2 k)_{+}-2(j-2 l-2 k-1)_{+}
\end{array}
$$

There are, again, two cases, depending on if $3 l+2 k \leq 2 l+3 k$ or $2 l+3 k \leq 3 l+2 k$. First, suppose $3 l+2 k \leq 2 l+3 k$.

$$
\begin{array}{ccccc}
0 \leq j \leq 2 l+2 k+1 & \Rightarrow & G \cdot C_{45} & = & j \geq 0 \\
2 l+2 k+1<j \leq 3 l+2 k & \Rightarrow & G \cdot C_{45} & = & -j+4 l+4 k+2 \geq 0 \\
3 l+2 k<j \leq 2 l+3 k & \Rightarrow & G \cdot C_{45} & = & -2 j+7 l+6 k+2 \geq 0 \\
2 l+3 k<j<3 l+3 k & \Rightarrow & G \cdot C_{45} & = & -3 j+9 l+9 k+2 \geq 0
\end{array}
$$

If $2 l+3 k \leq 3 l+2 k$, then the values differ for $j$ such that:

$$
\begin{aligned}
& 2 l+2 k+1<j \leq 2 l+3 k \Rightarrow G \cdot C_{45}=-j+4 l+4 k+2 \geq 0 \\
& 2 l+3 k<j \leq 3 l+2 k \quad \Rightarrow \quad G \cdot C_{45}=-2 j+6 l+7 k+2 \geq 0
\end{aligned}
$$

So $G \cdot C_{45} \geq 0$ when $j<3 l+3 k$. Next, we test $G$ against $E_{1}, E_{4}$ and $E_{7}$.

$$
\begin{array}{rlc}
G \cdot E_{1} & = & a_{1}-2 t_{1}-t_{2} \\
& =(j-\phi(1)+1)_{+}-2(j-\phi(1)-\phi(2)+2)_{+}-(j-\phi(1)-2 \phi(3)+1)_{+} \\
& = & (j-l-2 k)_{+}-2(j-2 l-3 k)_{+}-(j-3 l-2 k)_{+}
\end{array}
$$

First, suppose $2 l+3 k \leq 3 l+2 k$.

$$
\begin{aligned}
& 0 \leq j \leq l+2 k \quad \Rightarrow \quad G \cdot E_{1} \quad=\quad j \geq 0 \\
& l+2 k<j \leq 2 l+3 k \Rightarrow G \cdot E_{1}=j-l-2 k \geq 0 \\
& 2 l+3 k<j \leq 3 l+2 k \Rightarrow G \cdot E_{1}=-j+3 l+4 k \geq 0 \\
& 3 l+2 k<j<3 l+3 k \Rightarrow G \cdot E_{1}=-2 j+6 l+6 k \geq 0
\end{aligned}
$$

If $3 l+2 k \leq 2 l+3 k$, then the values differ for $j$ such that:

$$
\begin{aligned}
& l+2 k<j \leq 3 l+2 k \Rightarrow G \cdot E_{1}=j-l-2 k \geq 0 \\
& 3 l+2 k<j \leq 2 l+3 k \Rightarrow G \cdot E_{1} \quad \Rightarrow \quad 2 l \geq 0
\end{aligned}
$$

In both cases, we see that $G \cdot E_{1}<0$ if $2 j>6 l+6 k$ if $j \geq 3 l+3 k+1=\phi(1)+\phi(2)+$ $\phi(3)-1$. A similar analysis shows that $G \cdot E_{4}<0$ iff $j \geq \phi(1)+\phi(2)+\phi(3)-2$, and $G \cdot E_{7}<0$ iff $j \geq \phi(1)+\phi(2)+\phi(3)-1$. This concludes the proof if $\Delta=(k, k+1)$.

A similar argument yields the Hilbert function if $\Delta=(k+1, k)$; when expressed in terms of $k, l$ it differs from the formula for $\Delta=(k, k+1)$. But because

$$
\begin{array}{ccc}
\Delta=(k, k+1) \quad & \Rightarrow & 2 \phi(2)+\phi(3)=3 l+2 k+2 \\
\Delta=(k+1, k) & \Rightarrow \quad 2 \phi(2)+\phi(3)=3 l+2 k,
\end{array}
$$

writing the Hilbert function in terms of $\phi$ yields a formula independent of $\Delta$. 
Corollary 5.3. For $n=3$ and $\phi$ almost linear, if char $(\mathbb{K})>\phi(1)+\phi(2)+$ $\phi(3)-2$ or $\operatorname{char}(\mathbb{K})=0$, then $P\left(R / J_{\phi}, t\right)=$

$$
\frac{1-3 t^{\phi(1)}-3 t^{2 \phi(2)}-t^{3 \phi(3)}+3 t^{2 \phi(2)+\phi(3)}+3 t^{\phi(1)+2 \phi(3)}+6 t^{\phi(1)+\phi(2)}-6 t^{\phi(1)+\phi(2)+\phi(3)}}{(1-t)^{3}} \text {. }
$$

Example 5.4. Let $\operatorname{char}(\mathbb{K})=0$. The almost linear degree function

$$
\phi(1)=16, \phi(2)=12, \phi(3)=7,
$$

has $\Delta=(4,5)$ and associated Hilbert series

$$
\frac{1-3 t^{16}-t^{21}-3 t^{24}+6 t^{28}+3 t^{30}+3 t^{31}-6 t^{35}}{(1-t)^{3}}
$$

The almost linear degree function

$$
\phi(1)=20, \phi(2)=16, \phi(3)=13,
$$

has $\Delta=(4,3)$ and associated Hilbert series

$$
\frac{1-3 t^{20}-3 t^{32}+6 t^{36}-t^{39}+3 t^{45}+3 t^{46}-6 t^{49}}{(1-t)^{3}} .
$$

Postnikov and Shapiro note that when the degree function is not linear or almost linear, they were unable to find a case where the equality of Theorem 1.1 held. In general, Harbourne's methods can be applied to determine the Hilbert series of any ideal generated by powers of linear forms in three variables, as long as there are at most eight generators (and in certain cases, more).

\section{Examples, conjectures and a counterexample}

We assume throughout this section that $\operatorname{char}(\mathbb{K})>\phi(1)+\phi(2)+\phi(3)-2$ or $\operatorname{char}(\mathbb{K})=0$. We use $G_{\phi}$ to denote an ideal of generic forms generated in the same degrees as $J_{\phi}$.

Example 6.1. We revisit Example 3.3. For this example we have:

$$
P\left(R / G_{\phi}, t\right)=P\left(R / J_{\phi}, t\right)=P\left(R / I_{\phi}, t\right),
$$

the Hilbert series for all three is

$$
1+3 t+6 t^{2}+9 t^{3}+12 t^{4}+12 t^{5}+6 t^{6}
$$

Example 6.2. The degree function $\phi(1)=8, \phi(2)=5, \phi(3)=3$ is almost linear, but not linear. We illustrate the computation of $h^{0}\left(D_{13}\right)$. Since $t_{1}=2$ we first reduce to $D_{13}-2\left(C_{124}+C_{135}+C_{236}\right)$, yielding

$$
D^{\prime}=7 E_{0}-\sum_{i=1}^{6} 2 E_{i}-5 E_{7} .
$$

Finally, we check that $t_{2}=1$ (this is the almost linear $t_{2}$ ), so

$$
G=4 E_{0}-\sum_{i=1}^{6} E_{i}-2 E_{7}
$$


We find

$$
h^{0}(G)=\frac{G^{2}-G K_{X}}{2}+1=6 .
$$

Of course, Corollary 5.3 tells us that

$$
P\left(R / J_{\phi}, t\right)=\frac{1-t^{6}-3 t^{8}-3 t^{10}+9 t^{13}+3 t^{14}-6 t^{16}}{(1-t)^{3}}
$$

so this is the expected value.

We close the paper with a conjecture from [22] about the minimal free resolution of $J_{\phi}$ when $\phi$ is linear. Roughly speaking, the conjecture is that the resolutions are as simple as can be expected. In [20], Postnikov and Shapiro generalize this conjecture to a broader class of families.

Conjecture 6.3. For $n=3$ and $\phi$ linear, the minimal free resolution of $J_{\phi}$ is given by:

$$
0 \longrightarrow R^{6}\left(-\sum_{i=1}^{3} \phi(i)\right) \longrightarrow \begin{gathered}
R^{6}(-2 \phi(2)-\phi(3)) \\
\oplus \\
R^{6}(-\phi(1)-\phi(2))
\end{gathered} \longrightarrow \begin{gathered}
R^{3}(-\phi(1)) \\
\oplus \\
R^{3}(-2 \phi(2)) \\
\oplus \\
R(-3 \phi(3))
\end{gathered} \longrightarrow J_{\phi} \longrightarrow 0 .
$$

A similar pair of conjectures can be made for the almost linear case; when $\Delta=(k+1, k)$ the conjecture is the natural analog of Conjecture 6.3. However, interesting things happen when $\Delta=(k, k+1)$.

Example 6.4. For the almost linear degree function

$$
\phi(1)=8, \phi(2)=5, \phi(3)=1,
$$

the minimal free resolution of $J_{\phi}$ is given by:

$$
0 \rightarrow R^{6}(-14) \rightarrow R^{6}(-13) \oplus R^{3}(-11) \rightarrow R^{3}(-8) \oplus R(-3) \rightarrow J_{\phi} \rightarrow 0
$$

and the minimal free resolution of $I_{\phi}$ is given by:

$$
\begin{array}{ccc}
R^{6}(-13) & & R^{3}(-10) \\
\oplus & & \oplus \\
& \oplus R^{6}(-14) \rightarrow & R^{3}(-11) \\
\oplus & & R^{3}(-8) \\
R^{3}(-10) & & \oplus \\
\oplus(-3) & &
\end{array}
$$

So while $P\left(R / J_{\phi}, t\right)=P\left(R / I_{\phi}, t\right)$ as required by Corollary 5.3 , the minimal free resolutions differ. In particular, this gives a counterexample to Conjecture 6.10 of [20] (see also remarks after Corollary 12.2).

\section{Acknowledgments}

I thank Boris Shapiro for explaining the problem to me, M.F.I.-Oberwolfach for bringing us together, and Brian Harbourne and Tony Iarrobino for several enlightening conversations. The Macaulay 2 software package was also a help, as were comments from an anonymous referee. 


\section{References}

[1] D. Anick, Thin algebras of embedding dimension three, J. Algebra, 100 (1986), 235-259.

[2] A. Buckley and M. Zompatori, Linear systems of plane curves with a composite number of base points of equal multiplicity, Trans. Amer. Math. Soc., 355 (2003), 539-549.

[3] C. Ciliberto and R. Miranda, Linear systems of plane curves with base points of equal multiplicity, Trans. Amer. Math. Soc., 352 (2000), 4037-4050.

[4] D. Eisenbud, Commutative Algebra with a view towards Algebraic Geometry, SpringerVerlag, Berlin-Heidelberg-New York, 1995.

[5] J. Emsalem and A. Iarrobino, Inverse system of a symbolic power I, J. Algebra, 174 (1995), 1080-1090. 787-796.

[6] R. Fröberg, An inequality for Hilbert series of graded algebras, Math. Scand., 56 (1985), 117-144.

[7] A. Geramita, Inverse Systems of Fat Points: Waring's Problem, Secant Varieties of Veronese Varieties, and Parameter Spaces for Gorenstein Ideals, Queens Papers in Pure and Applied Mathematics 102 (1996), 1-114.

[8] B. Harbourne, Complete linear systems on rational surfaces, Trans. Amer. Math. Soc., 289 (1985), 213-226.

[9] _ Problems and progress: survey on fat points in $\mathbb{P}^{2}$, Queens Papers in Pure and Applied Mathematics 123 (2002), 85-132.

[10] _ Rational surfaces with $K^{2}>$ 0, Proc. Amer. Math. Soc., 124 (1996), 727-733.

[11] _ Anticanonical rational surfaces, Trans. Amer. Math. Soc., 349 (1997), 11911208.

[12] B. Harbourne and J. Roé, Linear systems with multiple base points in $\mathbb{P}^{2}$, Advances in Geometry, 4 (2004), 41-59.

[13] R. Hartshorne, Algebraic Geometry, Graduate Texts in Mathematics, 52, Springer-Verlag, Berlin-Heidelberg-New York, 1977. 319-356.

[14] A. Iarrobino, V. Kanev, Power sums, Gorenstein algebras, and determinantal loci, SLNM 1721. Springer-Verlag, Berlin, 1999.

[15] F.H.S. Macaulay, The algebraic theory of modular systems, Cambridge University Press, London/New York, 1916.

[16] Y. Manin, Cubic Forms: algebra, geometry, arithmetic, North Holland, New York, 1974.

[17] R. Miranda, Linear systems of plane curves, Notices Amer. Math. Soc., 46 (1999), 192201.

[18] M. Nagata, On rational surfaces. II, Mem. Coll. Sci. Univ. Kyoto Ser. A Math., 33 (1960), 271-293.

[19] A. Postnikov and B. Shapiro, Monotone monomial ideals and their equisupported deformations, preprint, March 2002.

[20] - Trees, Parking Functions, Syzygies, and Deformations of Monomial Ideals, Trans. Amer. Math. Soc., 356 (2004), 3109-3142.

[21] A. Postnikov, B. Shapiro, M. Shapiro, Algebras of curvature forms on homogeneous manifolds, AMS translations, Series 2194 (1999), 227-235.

[22] H. Schenck, Personal communication to B. Shapiro, April 2002.

[23] D. Taylor, Ideals generated by monomials in an R-sequence, Thesis, U. Chicago, (1966).

[24] A. Terracini, Sulle $V_{k}$ per cui la varieta degli $S_{h}(h+1)$-seganti ha dimensione minore dell'ordinario, Rend. Circ. Math. Palermo, 31 (1911), 527-530.

[25] _ Sulle rappresentazione delle coppie di forme ternarie mediante somme di potenze di forme lineari, Annali di Mat. Pura Appl. Serie III, 24 (1915), 1-10.

Department of Mathematics, Texas A\&M University, College Station, TX 77843

E-mail address: schenck@math.tamu.edu 